\title{
CONSTRUCTION OF THE NON-RIGID EARTH ROTATION
} SERIES

\author{
V.V. Pashkevich \\ Central (Pulkovo) Astronomical Observatory of RAS \\ Pulkovskoe shosse, 65/1, 196140, St. Petersburg, Russia \\ e-mails: apeks@gao.spb.ru
}

\begin{abstract}
Last years a lot of attempts to derive a high-precision theory of the non-rigid Earth rotation are carried out. For these purposes different transfer functions are used. Usually these transfer functions are applied to the series representing the nutation in the longitude and the obliquity of the rigid Earth rotation with respect to the ecliptic of date. The aim of this investigation is a construction of new high-precision non-rigid Earth rotation series (SN9000), dynamically adequate to the DE404/LE404 ephemeris over 2000 time span years, which are presented as functions of the Euler angles $\psi, \theta$ and $\varphi$ with respect to the fixed ecliptic plane and equinox J2000.0.
\end{abstract}

Keywords: non-rigid Earth rotation, transfer functions, ephemeris, least-squares method, spectral analysis.

\section{INTRODUCTION}

The early stages of the previous investigation:

1. The high-precision numerical solution of the rigid Earth rotation has been constructed by V.V.Pashkevich, G.I.Eroshkin and A.Brzezinski (2004), V.V.Pashkevich and G.I.Eroshkin (Proceedings of Journees 2004). The initial conditions have been calculated from SMART97 by P.Bretagnon, G.Francou, P.Rocher, J.L.Simon (1998). The discrepancies between the numerical solution and the semi-analytical solution SMART97 were obtained in the Euler angles over 2000 years time interval with one-day spacing.

2. The investigation of the discrepancies was carried out by the least squares and by the spectral analysis algorithms V.V.Pashkevich and G.I.Eroshkin (Proceedings of Journees 2005). The high-precision rigid Earth rotation series S9000 were determined by V.V.Pashkevich and G.I.Eroshkin (2005).

The next stage of this investigation:

3. The new high-precision non-rigid Earth rotation series (SN9000), which are presented as functions of the Euler angles, are constructed by using the method of P.Bretagnon, P.M.Mathews, J.L.Simon (1999) and the transfer function MHB2002 of Mathews, P. M., Herring, T. A., and Buffett B. A. (2002). 
Expressions for the Euler angles are:

$$
\begin{aligned}
& \left.\left.\begin{array}{l}
\psi=\psi_{p}+\Delta \psi \\
\theta=\theta_{p}+\Delta \theta \\
\varphi=\varphi_{p}+\Delta \varphi
\end{array}\right\}, \begin{array}{c}
\psi_{p}= \\
\theta_{p}=\theta_{0}+\theta_{1} t+\ldots+\theta_{5} t^{5} \\
\varphi_{p}=\varphi_{0}+\varphi_{1} t+\ldots+\varphi_{5} t^{5}
\end{array}\right\}, \quad \text { where } \psi_{0}=0 \\
& \left.\begin{array}{l}
\Delta \psi=\sum_{j} \sum_{k=0}^{4}\left[\Delta \psi_{j k}^{i p} \sin \left(v_{j 0}+v_{j 1} t\right)+\Delta \psi_{j k}^{o p} \cos \left(v_{j 0}+v_{j 1} t\right)\right] t^{k} \\
\Delta \theta=\sum_{j} \sum_{k=0}^{4}\left[\Delta \theta_{j k}^{o p} \sin \left(v_{j 0}+v_{j 1} t\right)+\Delta \theta_{j k}^{i p} \cos \left(v_{j 0}+v_{j 1} t\right)\right] t^{k} \\
\Delta \varphi=\sum_{j} \sum_{k=0}^{4}\left[\Delta \varphi_{S j k} \sin \left(v_{j 0}+v_{j 1} t\right)+\Delta \varphi_{C j k} \cos \left(v_{j 0}+v_{j 1} t\right)\right] t^{k}
\end{array}\right\} .
\end{aligned}
$$

Here the quantities with p-index are the precessional parts of the Euler angles; the quantities with $\Delta$ are the nutational parts of the Euler angles; $\mathrm{t}$ is the time; $v_{j 0}+v_{j 1} t$ is the argument of nutation; $\Delta \psi_{j k}^{i p}, \Delta \theta_{j k}^{i p}$ are the amplitudes of the in-phase terms; $\Delta \psi_{j k}^{o p}, \Delta \theta_{j k}^{o p}$ are the amplitudes of the out-of-phase terms; $\Delta \varphi_{S j k}, \Delta \varphi_{C j k}$ are the amplitudes of sine and cosine terms of the proper rotation angle.

\section{CLASSICAL ALGORITHM}

The classical scheme for the construction of the non-rigid Earth rotation theories is defined only by two Euler angels - the longitude and the obliquity. In it, some transfer functions are applied to the series representing the nutation in the longitude and the obliquity of the rigid Earth rotation with respect to the ecliptic of date. Usually the expressions for the nutation and the transfer function are presented in the complex form:

$$
\left.\begin{array}{c}
\zeta(t)=\Delta \psi_{N R} \sin \theta_{0}+i \Delta \theta_{N R}, \quad z(t)=\Delta \psi_{R} \sin \theta_{0}+i \Delta \theta_{R} \\
\zeta(t)=g(\sigma) z(t), \quad g(\sigma)=g_{R E}(\sigma)+i g_{I M}(\sigma)
\end{array}\right\} \Rightarrow
$$

Here $\Delta \psi$ and $\Delta \theta$ are nutations in the longitude and the obliquity with respect to the ecliptic of date, respectively, for the non-rigid (the subscript "NR") and rigid (the subscript "R") Earth; $z(t)$ and $\zeta(t)$ are nutations of the rigid and the non-rigid Earth, respectively; $g(\sigma)$ is the transfer function; $\sigma=\frac{v_{j 1}}{\Omega_{0}}-1 ; v_{j 1}$ is the frequency of nutation; $i=\sqrt{-1}$; the subscript "RE" mean real parts of the complex values, the subscript "IM" mean imaginary parts of the complex values; $\Omega_{0}$ is the mean angular velocity of the Earth rotation $\left(\Omega_{0}=7.292115 \cdot 10^{-5} \mathrm{rad}\right.$ per s). 
Expressions for the non-rigid Earth nutations in the longitude and the obliquity, presented in (2), in the paper of Bretagnon et al. (1999) contain only the real parts of the complex values. The exact expressions for the non-rigid Earth nutations in the longitude and the obliquity, in the complex values, are obtained in this investigation:

$$
\begin{aligned}
& \Delta \psi_{N R} \sin \theta_{0}=\frac{1}{2} \sum_{k=1}^{4} t^{k} \sum_{j} {\left[\left(\Delta \psi_{R_{j k}}^{i p} \sin \theta_{0}\left(g_{R E}^{+}+g_{R E}^{-}\right)-s_{j} \Delta \theta_{R_{j k}}^{i p}\left(g_{R E}^{+}-g_{R E}^{-}\right)\right) \sin \Phi_{j}\right.} \\
&+\left(\Delta \psi_{R_{j k}}^{o p} \sin \theta_{0}\left(g_{R E}^{+}+g_{R E}^{-}\right)+s_{j} \Delta \theta_{R_{j k}}^{o p}\left(g_{R E}^{+}-g_{R E}^{-}\right)\right) \cos \Phi_{j} \\
&+\left(-s_{j} \Delta \psi_{R_{j k}}^{o p} \sin \theta_{0}\left(g_{I M}^{+}-g_{I M}^{-}\right)-\Delta \theta_{R_{j k} p}^{o p}\left(g_{I M}^{+}+g_{I M}^{-}\right)\right) \sin \Phi_{j} \\
&+\left.\left(s_{j} \Delta \psi_{R_{j k}}^{i p} \sin \theta_{0}\left(g_{I M}^{+}-g_{I M}^{-}\right)-\Delta \theta_{R_{j k}^{i p}}\left(g_{I M}^{+}+g_{I M}^{-}\right)\right) \cos \Phi_{j}\right] \\
& \Delta \theta_{N R}=\frac{1}{2} \sum_{k=1}^{4} t^{k} \sum_{j}\left[\left(s_{j} \Delta \psi_{R_{j k}}^{o p} \sin \theta_{0}\left(g_{R E}^{+}-g_{R E}^{-}\right)+\Delta \theta_{R_{j k}}^{o p}\left(g_{R E}^{+}+g_{R E}^{-}\right)\right) \sin \Phi_{j}\right. \\
&+\left(-s_{j} \Delta \psi_{R_{j k}}^{i p} \sin \theta_{0}\left(g_{R E}^{+}-g_{R E}^{-}\right)+\Delta \theta_{R_{j k} p}^{i p}\left(g_{R E}^{+}+g_{R E}^{-}\right)\right) \cos \Phi_{j} \\
&+\left(\Delta \psi_{R_{j k}}^{i p} \sin \theta_{0}\left(g_{I M}^{+}+g_{I M}^{-}\right)-s_{j} \Delta \theta_{R_{j k}}^{i p}\left(g_{I M}^{+}-g_{I M}^{-}\right)\right) \sin \Phi_{j} \\
&\left.+\left(\Delta \psi_{R_{j k}}^{o p} \sin \theta_{0}\left(g_{I M}^{+}+g_{I M}^{-}\right)+s_{j} \Delta \theta_{R_{j k}}^{o p}\left(g_{I M}^{+}-g_{I M}^{-}\right)\right) \cos \Phi_{j}\right]
\end{aligned}
$$

where $\Phi_{j}=v_{j 0}+v_{j 1} t ; s_{j}=\frac{\left|v_{j 1}\right|}{v_{j 1}} ; g^{+}=g\left(\left|\frac{v_{j 1}}{\Omega_{0}}\right|-1\right), g^{-}=g\left(-\left|\frac{v_{j 1}}{\Omega_{0}}\right|-1\right)$ are the transfer

functions for the prograde and retrograde frequencies, respectively.

\section{TRANSFER FUNCTIONS}

The types of the transfer functions, which are usually used in different researches, are following:

a) J.M.Wahr (1981), V. Dehant and P. Defraigne (1997):

$$
\begin{aligned}
& g(\sigma)=1+\left(a_{O 1}+a_{C W} \frac{\sigma-\lambda_{O 1}}{\lambda_{C W}-\sigma}+a_{F C N} \frac{\sigma-\lambda_{O 1}}{\lambda_{F C N}-\sigma}+a_{C W R} \frac{\sigma-\lambda_{O 1}}{\lambda_{C W R}-\sigma}\right)\left(\lambda_{T O M}-\sigma\right)\left(\sigma-\lambda_{C W R}\right), \\
& g(-1)=1
\end{aligned}
$$

where the coefficients $a_{C W}, a_{C W R}, a_{F C N}, a_{O 1}$ and the frequencies $\lambda_{T O M}, \lambda_{C W}, \lambda_{C W R}, \lambda_{F C N}, \lambda_{O 1}$ are given by the different models.

b) T. Shirai and T. Fukushima (2001):

$$
\begin{aligned}
& g(\hat{n})=R+R^{\prime} \hat{n}+\sum_{j=1}^{2} \frac{R_{j}}{\hat{n}-\hat{n}_{j}}, \\
& g(0)=1 \quad \text { (4a) }
\end{aligned}
$$

where the coefficients $R_{j}(j=1,2), R, R^{\prime}$ are complex numbers, $\hat{n}=\sigma+1=\frac{v_{j 1}}{\Omega_{0}} ; \hat{n}_{j}$ are two 
resonance frequencies (Free Core Nutation and Chandler wobble). Parameters of Nutation are taken from different models. The properties of the "gyrostatic rigidity" in (4) and (4a) mean that the transfer function does not change the constant term.

c) P.Bretagnon, P.M. Mathews, J.-L. Simon (1999):

$$
T\left(\sigma ; e \mid e_{R}\right)=\frac{\left(e_{R}-\sigma\right)}{\left(e_{R}+1\right)} N_{0}\left(1+(1+\sigma) \sum_{\alpha} \frac{N_{\alpha}}{\sigma-\sigma_{\alpha}}\right) .
$$

d) P.M. Mathews, T.A. Herring, B.A. Buffett (2002):

$$
\begin{aligned}
& T\left(\sigma ; e \mid e_{R}\right)=\frac{\left(e_{R}-\sigma\right)}{\left(e_{R}+1\right)} N_{0}\left[1+(1+\sigma)\left(Q_{0}+\sum_{\alpha=1}^{4} \frac{Q_{\alpha}}{\sigma-s_{\alpha}}\right)\right], \\
& T\left(-1 ; e \mid e_{R}\right)=N_{0}, \text { where } N_{0}=\frac{e /(1+e)}{e_{R} /\left(1+e_{R}\right)} \approx 1
\end{aligned}
$$

Here $e_{R}, e$ represent the ellipticities for the rigid and non-rigid Earth, respectively; $\hat{n}=\sigma+1$ is the angular frequency, which is measured in cycles per sidereal day (cpsd) in space; the complex coefficients $N_{\alpha}(\alpha=1, \ldots, 4), Q_{\alpha}(\alpha=0, \ldots, 4)$ and the complex frequencies $\sigma_{\alpha}(\alpha=1, \ldots, 4)$, $s_{\alpha}(\alpha=1, \ldots, 4)$ are given by the different models.

\section{ALGORITHM OF BRETAGNON ET AL. (1999)}

In order to construct the non-rigid Earth rotation theory for all three Euler angles, the transfer function is applied to the rigid Earth angular velocity vector. It is realized in the algorithm of Bretagnon et al. (1999).

\section{STEP 1 - Representation of the rigid Earth angular velocity vector}

The components of the rigid Earth angular velocity vector have a form:

$$
\begin{aligned}
& p_{R}=\dot{\psi}_{R} \sin \theta_{R} \sin \varphi_{R}+\dot{\theta}_{R} \cos \varphi_{R}, \\
& q_{R}=\dot{\psi}_{R} \sin \theta_{R} \cos \varphi_{R}-\dot{\theta}_{R} \sin \varphi_{R}, \\
& r_{R}=\dot{\psi}_{R} \cos \theta_{R}+\dot{\varphi}_{R} .
\end{aligned}
$$

Here and further a dot over a letter means the differentiation with respect to time, $\psi, \theta$ and $\varphi$ are Euler angles with respect to the fixed ecliptic plane and equinox J2000.0.

Expressions for the components of the Earth's angular velocity vector are following: 


$$
\left.\begin{array}{l}
p=\sum_{j} \sum_{k=0}^{4}\left[p_{S_{j k}} \sin \left(v_{j 0}+v_{j 1} t\right)+p_{C_{j k}} \cos \left(v_{j 0}+v_{j 1} t\right)\right] t^{k} \\
q=\sum_{j} \sum_{k=0}^{4}\left[q_{S_{j k}} \sin \left(v_{j 0}+v_{j 1} t\right)+q_{C_{j k}} \cos \left(v_{j 0}+v_{j 1} t\right)\right] t^{k} \\
r=\sum_{j} \sum_{k=0}^{4}\left[r_{S_{j k}} \sin \left(v_{j 0}+v_{j 1} t\right)+r_{C_{j k}} \cos \left(v_{j 0}+v_{j 1} t\right)\right] t^{k}
\end{array}\right\},
$$

where $p_{C_{j k}}, q_{C_{j k}}, r_{C_{j k}}$ are the secular terms in these components of $v_{j 0}=0$ and $v_{j 1}=0 ; j$ is the number of terms, which are included in the described series.

The coefficients $p_{C_{j k}}, p_{S_{j k}}, q_{C_{j k}}, q_{S_{j k}}$ contain in-phase components and out-of-phase components: $p_{C_{j k}}=p_{C_{j k}}^{i p}+p_{C_{j k}}^{o p} ; p_{S_{j k}}=p_{S_{j k}}^{i p}+p_{S_{j k}}^{o p} ; q_{C_{j k}}=q_{C_{j k}}^{i p}+q_{C_{j k}}^{o p} ; q_{S_{j k}}=q_{S_{j k}}^{i p}+q_{S_{j k}}^{o p}$, where $i p$ is in phase, $o p$ is out of phase.

The parts of the components of the rigid Earth angular velocity vector are following:

$$
\left.\begin{array}{l}
\dot{\psi}_{j k} \sin \theta=\dot{\psi}_{j k}^{i p} \sin \theta \sin \left(v_{j 0}+v_{j 1} t\right)+\dot{\psi}_{j k}^{o p} \sin \theta \cos \left(v_{j 0}+v_{j 1} t\right) \\
\dot{\theta}_{j k}=\dot{\theta}_{j k}^{o p} \sin \left(v_{j 0}+v_{j 1} t\right)+\dot{\theta}_{j k}^{i p} \cos \left(v_{j 0}+v_{j 1} t\right) \\
\dot{\varphi}_{j k}=\dot{\varphi}_{j k}^{S} \sin \left(v_{j 0}+v_{j 1} t\right)+\dot{\varphi}_{j k}^{C} \cos \left(v_{j 0}+v_{j 1} t\right)
\end{array}\right\}
$$

The transformation of the arguments of the components of the rigid Earth angular velocity vector can be derived as follows:

$$
\begin{aligned}
\text { Let } v_{j 1}=s_{j}\left|v_{j 1}\right| \Rightarrow s_{j}= & \frac{\left|v_{j 1}\right|}{v_{j 1}} \Rightarrow v_{j 0}+v_{j 1} t=s_{j}\left(\left|v_{j 1}\right| t+s_{j} v_{j 0}\right)=s_{j} \chi_{j} \Rightarrow \chi_{j}=\left|v_{j 1}\right| t+s_{j} v_{j 0} \\
& \left.\Rightarrow \begin{array}{l}
\dot{\psi}_{j k} \sin \theta=s_{j} \dot{\psi}_{j k}^{i p} \sin \theta \sin \chi_{j}+\dot{\psi}_{j k}^{o p} \sin \theta \cos \chi_{j} \\
\dot{\theta}_{j k}=\dot{\theta}_{j k}^{i p} \cos \chi_{j}+s_{j} \dot{\theta}_{j k}^{o p} \sin \chi_{j}
\end{array}\right\} .(7 \mathrm{a})
\end{aligned}
$$

The components of the coefficients $p$ and $q$ are presented in the complex form $(p, q)$. The components of the coefficients $p$ and $q$ include the prograde and retrograde parts: 


$$
\left.\begin{array}{l}
\left(p_{S_{j k}^{i p}}^{i p}, q_{S_{j k}^{i p}}^{i p}\right)=\left(s_{j} \dot{\psi}_{j k}^{i p} \sin \theta \sin \chi_{j},-\dot{\theta}_{j k}^{i p} \cos \chi_{j}\right) \sin \varphi \\
\left(p_{C_{j k}}^{i p}, q_{C_{j k}}^{i p}\right)=\left(\begin{array}{ll}
\dot{\theta}_{j k}^{i p} \cos \chi_{j}, & \left.s_{j} \dot{\psi}_{j k}^{i p} \sin \theta \sin \chi_{j}\right) \cos \varphi \\
\left(p_{S_{j k}}^{o p}, q_{S_{j k}}^{o p}\right)=\left(\dot{\psi}_{j k}^{o p} \sin \theta \cos \chi_{j},-s_{j} \dot{\theta}_{j k}^{o p} \sin \chi_{j}\right) \sin \varphi \\
\left(p_{C_{j k}}^{o p}, q_{C_{j k}}^{o p}\right)=\left(s_{j} \dot{\theta}_{j k}^{o p} \sin \chi_{j}, \quad \dot{\psi}_{j k}^{o p} \sin \theta \cos \chi_{j}\right) \cos \varphi
\end{array}\right\} \Rightarrow, \\
\left(p_{S_{j k}}^{i p}, q_{S_{j k}}^{i p}\right)=B_{j k}^{P R O i p}\left(\sin \chi_{j},-\cos \chi_{j}\right)+B_{j k}^{R E T i p}\left(-\sin \chi_{j},-\cos \chi_{j}\right) \\
\left(p_{C_{j k}}^{i p}, q_{C_{j k}}^{i p}\right)=C_{j k}^{P R O i p}\left(\cos \chi_{j}, \sin \chi_{j}\right)+C_{j k}^{R E T i p}\left(\cos \chi_{j},-\sin \chi_{j}\right) \\
\left(p_{S_{j k}^{o p}}^{o p}, q_{S_{j k}}^{o p}\right)=B_{j k}^{P R O o p}\left(\cos \chi_{j}, \sin \chi_{j}\right)+B_{j k}^{R E T o p}\left(\cos \chi_{j},-\sin \chi_{j}\right) \\
\left(p_{C_{j k}}^{o p}, q_{C_{j k}}^{o p}\right)=C_{j k}^{P R O o p}\left(\sin \chi_{j},-\cos \chi_{j}\right)+C_{j k}^{R E T o p}\left(-\sin \chi_{j},-\cos \chi_{j}\right)
\end{array}\right\},
$$

where $P R O$ is Prograde, $R E T$ is Retrograde.

The amplitudes of the prograde and retrograde components are following:

$$
\begin{aligned}
& B_{j k}^{\text {PROip }}=\frac{1}{2}\left(s_{j} \dot{\psi}_{j k}^{i p} \sin \theta+\dot{\theta}_{j k}^{i p}\right) \sin \varphi \| C_{j k}^{P R O i p}=\frac{1}{2}\left(\dot{\theta}_{j k}^{i p}+s_{j} \dot{\psi}_{j k}^{i p} \sin \theta\right) \cos \varphi \\
& B_{j k}^{R E T i p}=-\frac{1}{2}\left(s_{j} \dot{\psi}_{j k}^{i p} \sin \theta-\dot{\theta}_{j k}^{i p}\right) \sin \varphi \mid C_{j k}^{R E T i p}=\frac{1}{2}\left(\dot{\theta}_{j k}^{i p}-s_{j} \dot{\psi}_{j k}^{i p} \sin \theta\right) \cos \varphi \\
& B_{j k}^{P R O o p}=\frac{1}{2}\left(\dot{\psi}_{j k}^{o p} \sin \theta-s_{j} \dot{\theta}_{j k}^{o p}\right) \sin \varphi \mid C_{j k}^{P R O o p}=\frac{1}{2}\left(s_{j} \dot{\theta}_{j k}^{o p}-\dot{\psi}_{j k}^{o p} \sin \theta\right) \cos \varphi \\
& B_{j k}^{R E T o p}=\frac{1}{2}\left(\dot{\psi}_{j k}^{o p} \sin \theta+s_{j} \dot{\theta}_{j k}^{o p}\right) \sin \varphi \| C_{j k}^{R E T o p}=-\frac{1}{2}\left(s_{j} \dot{\theta}_{j k}^{o p}+\dot{\psi}_{j k}^{o p} \sin \theta\right) \cos \varphi
\end{aligned}
$$

\section{STEP 2 - Construction of the non-rigid Earth angular velocity vector}

In this section new formulas are obtained for the complex transformation form of the components of the rigid angular velocity vector to the components of the non-rigid angular velocity vector.

The components of the non-rigid Earth angular velocity vector are:

$$
\left(p_{N R}+i q_{N R}\right)=\left(p_{R}+i q_{R}\right) g(\sigma) .
$$

Let us assume that $r_{N R}=r_{R}$, as in the paper of Bretagnon et al. (1999). It is the classical method. The full method is to take into account the tidal variations in the Earth's rotation: $r_{N R}=r_{R}+$ tidal variations.

The complex transformation of the components of the angular velocity vector is following:

$$
\begin{aligned}
& \left(p_{N R}, q_{N R}\right)=\left(p_{R}, q_{R}\right)\left(g_{R E}, g_{I M}\right)=\left(p_{R} g_{R E}-q_{R} g_{I M}, p_{R} g_{I M}+q_{R} g_{R E}\right), \\
& \text { where } g(\sigma)=\left(g_{R E}, g_{I M}\right) .
\end{aligned}
$$

The prograde and retrograde components of the coefficients $p$ and $q$ in the case of the nonrigid Earth can be expressed as: 


$$
\left.\begin{array}{l}
\left(p_{N S_{j k}}^{\text {PROip }}, q_{N S_{j k}}^{\text {PROip }}\right)=B_{j k}^{\text {PROip }}\left(\sin \chi_{j},-\cos \chi_{j}\right)\left(g_{R E}^{+}, g_{I M}^{+}\right) \\
\left(p_{N C_{j k}}^{\text {PROip }}, q_{N C_{j k}}^{\text {PROip }}\right)=C_{j k}^{\text {PROip }}\left(\cos \chi_{j}, \sin \chi_{j}\right)\left(g_{R E}^{+}, g_{I M}^{+}\right) \\
\left(p_{N S_{j k}}^{P R O o p}, q_{N S_{j k}}^{\text {PROop }}\right)=B_{j k}^{\text {PROop }}\left(\cos \chi_{j}, \sin \chi_{j}\right)\left(g_{R E}^{+}, g_{I M}^{+}\right) \\
\left(p_{N C_{j k}}^{P R O o p}, q_{N C_{j k}}^{P R O o p}\right)=C_{j k}^{P R O o p}\left(\sin \chi_{j},-\cos \chi_{j}\right)\left(g_{R E}^{+}, g_{I M}^{+}\right)
\end{array}\right\},
$$

where $g^{+}=g(|\hat{n}|-1), g^{-}=g(-|\hat{n}|-1)$ are the transfer functions for the prograde and retrograde frequencies, respectively.

The prograde and retrograde components of the coefficients of $p, q$ in (6) in the case of the non-rigid Earth are following:

$$
\begin{aligned}
& p_{N S_{j k}}^{P R O}=\frac{1}{2}\left[\left(\dot{\psi}_{j k}^{i p} \sin \theta+s_{j} \dot{\theta}_{j k}^{i p}\right)\left(g_{R E}^{+} \sin \varphi-g_{I M}^{+} \cos \varphi\right)+\left(-s_{j} \dot{\psi}_{j k}^{o p} \sin \theta+\dot{\theta}_{j k}^{o p}\right)\left(g_{I M}^{+} \sin \varphi+g_{R E}^{+} \cos \varphi\right)\right], \\
& p_{N S_{j k}}^{R E T}= \frac{1}{2}\left[\left(\dot{\psi}_{j k}^{i p} \sin \theta-s_{j} \dot{\theta}_{j k}^{i p}\right)\left(g_{R E}^{-} \sin \varphi-g_{I M}^{-} \cos \varphi\right)+\left(s_{j} \dot{\psi}_{j k}^{o p} \sin \theta+\dot{\theta}_{j k}^{o p}\right)\left(g_{I M}^{-} \sin \varphi+g_{R E}^{-} \cos \varphi\right)\right], \\
& p_{N C_{j k}}^{P R O}= \frac{1}{2}\left[\left(s_{j} \dot{\psi}_{j k}^{i p} \sin \theta+\dot{\theta}_{j k}^{i p}\right)\left(g_{I M}^{+} \sin \varphi+g_{R E}^{+} \cos \varphi\right)+\left(\dot{\psi}_{j k}^{o p} \sin \theta-s_{j} \dot{\theta}_{j k}^{o p}\right)\left(g_{R E}^{+} \sin \varphi-g_{I M}^{+} \cos \varphi\right)\right], \\
& p_{N C_{j k}}^{R E T}= \frac{1}{2}\left[\left(-s_{j} \dot{\psi}_{j k}^{i p} \sin \theta+\dot{\theta}_{j k}^{i p}\right)\left(g_{I M}^{-} \sin \varphi+g_{R E}^{-} \cos \varphi\right)+\left(\dot{\psi}_{j k}^{o p} \sin \theta+s_{j} \dot{\theta}_{j k}^{o p}\right)\left(g_{R E}^{-} \sin \varphi-g_{I M}^{-} \cos \varphi\right)\right], \\
& q_{N S_{j k}}^{P R O}=\frac{1}{2}\left[\left(\dot{\psi}_{j k}^{i p} \sin \theta+s_{j} \dot{\theta}_{j k}^{i p}\right)\left(g_{I M}^{+} \sin \varphi+g_{R E}^{+} \cos \varphi\right)+\left(s_{j} \dot{\psi}_{j k}^{o p} \sin \theta-\dot{\theta}_{j k}^{o p}\right)\left(g_{R E}^{+} \sin \varphi-g_{I M}^{+} \cos \varphi\right)\right], \\
& q_{N S_{j k}}^{R E T}=\frac{1}{2}\left[\left(\dot{\psi}_{j k}^{i p} \sin \theta-s_{j} \dot{\theta}_{j k}^{i p}\right)\left(g_{I M}^{-} \sin \varphi+g_{R E}^{-} \cos \varphi\right)+\left(-s_{j} \dot{\psi}_{j k}^{o p} \sin \theta-\dot{\theta}_{j k}^{o p}\right)\left(g_{R E}^{-} \sin \varphi-g_{I M}^{-} \cos \varphi\right)\right], \\
& q_{N C_{j k} P R O}^{P R}=\frac{1}{2}\left[\left(-s_{j} \dot{\psi}_{j k}^{i p} \sin \theta-\dot{\theta}_{j k}^{i p}\right)\left(g_{R E}^{+} \sin \varphi-g_{I M}^{+} \cos \varphi\right)+\left(\dot{\psi}_{j k}^{o p} \sin \theta-s_{j} \dot{\theta}_{j k}^{o p}\right)\left(g_{I M}^{+} \sin \varphi+g_{R E}^{+} \cos \varphi\right)\right], \\
& q_{N C_{j k}}^{R E T}=\frac{1}{2}\left[\left(s_{j} \dot{\psi}_{j k}^{i p} \sin \theta-\dot{\theta}_{j k}^{i p}\right)\left(g_{R E}^{-} \sin \varphi-g_{I M}^{-} \cos \varphi\right)+\left(\dot{\psi}_{j k}^{o p} \sin \theta+s_{j} \dot{\theta}_{j k}^{o p}\right)\left(g_{I M}^{-} \sin \varphi+g_{R E}^{-} \cos \varphi\right)\right] .
\end{aligned}
$$

The sine coefficients for the non-rigid Earth angular velocity vector can be derived after the summation of the prograde and retrograde parts: 


$$
\begin{aligned}
& p_{N S_{j k}}=\frac{1}{2}\left[\left(\dot{\psi}_{j k}^{i p} \sin \theta\left(g_{R E}^{+}+g_{R E}^{-}\right)+s_{j} \dot{\theta}_{j k}^{i p}\left(g_{R E}^{+}-g_{R E}^{-}\right)\right) \sin \varphi+\right. \\
& \left(-\dot{\psi}_{j k}^{i p} \sin \theta\left(g_{I M}^{+}+g_{I M}^{-}\right)-s_{j} \dot{\theta}_{j k}^{i p}\left(g_{I M}^{+}-g_{I M}^{-}\right)\right) \cos \varphi+ \\
& \left(-S_{j} \dot{\psi}_{j k}^{o p} \sin \theta\left(g_{I M}^{+}-g_{I M}^{-}\right)+\dot{\theta}_{j k}^{o p}\left(g_{I M}^{+}+g_{I M}^{-}\right)\right) \sin \varphi+ \\
& \left.\left(-S_{j} \dot{\psi}_{j k}^{o p} \sin \theta\left(g_{R E}^{+}-g_{R E}^{-}\right)+\dot{\theta}_{j k}^{o p}\left(g_{R E}^{+}+g_{R E}^{-}\right)\right) \cos \varphi\right], \\
& q_{N S_{j k}}=\frac{1}{2}\left[\left(\dot{\psi}_{j k}^{i p} \sin \theta\left(g_{I M}^{+}+g_{I M}^{-}\right)+s_{j} \dot{\theta}_{j k}^{i p}\left(g_{I M}^{+}-g_{I M}^{-}\right)\right) \sin \varphi+\right. \\
& \left(\dot{\psi}_{j k}^{i p} \sin \theta\left(g_{R E}^{+}+g_{R E}^{-}\right)+s_{j} \dot{\theta}_{j k}^{i p}\left(g_{R E}^{+}-g_{R E}^{-}\right)\right) \cos \varphi+ \\
& \left(s_{j} \dot{\psi}_{j k}^{o p} \sin \theta\left(g_{R E}^{+}-g_{R E}^{-}\right)-\dot{\theta}_{j k}^{o p}\left(g_{R E}^{+}+g_{R E}^{-}\right)\right) \sin \varphi+ \\
& \left.\left(-S_{j} \dot{\psi}_{j k}^{o p} \sin \theta\left(g_{I M}^{+}-g_{I M}^{-}\right)+\dot{\theta}_{j k}^{o p}\left(g_{I M}^{+}+g_{I M}^{-}\right)\right) \cos \varphi\right], \\
& r_{N S_{j k}}=\dot{\psi}_{j k}^{i p} \cos \theta+\dot{\varphi}_{j k}^{S} \text {, }
\end{aligned}
$$

where $p_{N S_{j k}}=p_{N S_{j k}}^{P R O}+p_{N S_{j k}}^{R E T} ; q_{N S_{j k}}=q_{N S_{j k}}^{P R O}+q_{N S_{j k}}^{R E T}$.

The cosine coefficients for the non-rigid Earth angular velocity vector can be derived after the summation of the prograde and retrograde parts:

$$
\begin{aligned}
& p_{N C_{j k}}=\frac{1}{2}\left[\left(s_{j} \dot{\psi}_{j k}^{i p} \sin \theta\left(g_{I M}^{+}-g_{I M}^{-}\right)+\dot{\theta}_{j k}^{i p}\left(g_{I M}^{+}+g_{I M}^{-}\right)\right) \sin \varphi+\right. \\
& \left(s_{j} \dot{\psi}_{j k}^{i p} \sin \theta\left(g_{R E}^{+}-g_{R E}^{-}\right)+\dot{\theta}_{j k}^{i p}\left(g_{R E}^{+}+g_{R E}^{-}\right)\right) \cos \varphi+ \\
& \left(\dot{\psi}_{j k}^{o p} \sin \theta\left(g_{R E}^{+}+g_{R E}^{-}\right)-s_{j} \dot{\theta}_{j k}^{o p}\left(g_{R E}^{+}-g_{R E}^{-}\right)\right) \sin \varphi+ \\
& \left.\left(-\dot{\psi}_{j k}^{o p} \sin \theta\left(g_{I M}^{+}+g_{I M}^{-}\right)+s_{j} \dot{\theta}_{j k}^{o p}\left(g_{I M}^{+}-g_{I M}^{-}\right)\right) \cos \varphi\right], \\
& q_{N C_{j k}}=\frac{1}{2}\left[\left(-S_{j} \dot{\psi}_{j k}^{i p} \sin \theta\left(g_{R E}^{+}-g_{R E}^{-}\right)-\dot{\theta}_{j k}^{i p}\left(g_{R E}^{+}+g_{R E}^{-}\right)\right) \sin \varphi+\right. \\
& \left(s_{j} \dot{\psi}_{j k}^{i p} \sin \theta\left(g_{I M}^{+}-g_{I M}^{-}\right)+\dot{\theta}_{j k}^{i p}\left(g_{I M}^{+}+g_{I M}^{-}\right)\right) \cos \varphi+ \\
& \left(\dot{\psi}_{j k}^{o p} \sin \theta\left(g_{I M}^{+}+g_{I M}^{-}\right)-s_{j} \dot{\theta}_{j k}^{o p}\left(g_{I M}^{+}-g_{I M}^{-}\right)\right) \sin \varphi+ \\
& \left.\left(\dot{\psi}_{j k}^{o p} \sin \theta\left(g_{R E}^{+}+g_{R E}^{-}\right)-s_{j} \dot{\theta}_{j k}^{o p}\left(g_{R E}^{+}-g_{R E}^{-}\right)\right) \cos \varphi\right], \\
& r_{N C_{j k}}=\dot{\psi}_{j k}^{o p} \cos \theta+\dot{\varphi}_{j k}^{C},
\end{aligned}
$$

where $p_{N C_{j k}}=p_{N C_{j k}}^{P R O}+p_{N C_{j k}}^{R E T} ; q_{N C_{j k}}=q_{N C_{j k}}^{P R O}+q_{N C_{j k}}^{R E T}$.

\section{STEP 3 - Calculation of the Euler angles for the case of the non-rigid Earth}

The term of the periodic parts for each of the Euler angles has same forms: 


$$
\begin{aligned}
x & =\sum_{k=0}^{m}\left[A_{k} \sin \left(c_{0}+c_{1} t\right)+B_{k} \cos \left(c_{0}+c_{1} t\right)\right] t^{k}, \\
\dot{x} & =\sum_{k=1}^{m}\left[\left(k A_{k}-c_{1} B_{k-1}\right) \sin \left(c_{0}+c_{1} t\right)+\left(k B_{k}+c_{1} A_{k-1}\right) \cos \left(c_{0}+c_{1} t\right)\right] t^{k-1} \\
& +c_{1}\left[-B_{m} \sin \left(c_{0}+c_{1} t\right)+A_{m} \cos \left(c_{0}+c_{1} t\right)\right] t^{m},
\end{aligned}
$$

Here $\mathrm{m}=1, \ldots, 4$ and $x=\Delta \psi_{j}, \Delta \theta_{j}, \Delta \varphi_{j}, \dot{x}=\Delta \dot{\psi}_{j}, \Delta \dot{\theta}_{j}, \Delta \dot{\varphi}_{j}$ represent the periodic part in each $\mathrm{j}$-th component of the Euler angels and their derivatives, respectively.

The values of the coefficients in $x$ can be computed by the following "Cascade" method:

$$
\begin{aligned}
& k A_{k}-c_{1} B_{k-1}=\alpha_{k-1}, k B_{k}+c_{1} A_{k-1}=\beta_{k-1}(\text { for } k=1, \ldots, m) \\
& -c_{1} B_{m}=\alpha_{m}, c_{1} A_{m}=\beta_{m} \Rightarrow A_{m}=\beta_{m} / c_{1}, B_{m}=-\alpha_{m} / c_{1} \\
& \Rightarrow A_{k-1}=\left(\beta_{k-1}-k B_{k}\right) / c_{1}, B_{k-1}=\left(k A_{k}-\alpha_{k-1}\right) / c_{1}(\text { for } k=m, \ldots, 1) .
\end{aligned}
$$

From the components of the non-rigid Earth angular velocity vector the derivatives of the Euler angles for the non-rigid Earth rotation are computed:

$$
\begin{aligned}
& \dot{\psi}_{N R}=\left(p_{N R} \sin \varphi_{N R}+q_{N R} \cos \varphi_{N R}\right) / \sin \theta_{N R}, \\
& \dot{\theta}_{N R}=p_{N R} \cos \varphi_{N R}-q_{N R} \sin \varphi_{N R}, \\
& \dot{\varphi}_{N R}=r_{N R}-\dot{\psi}_{N R} \cos \theta_{N R} .
\end{aligned}
$$

The periodic part of each $\mathrm{j}$-th component can be expressed as:

$$
\begin{aligned}
& \Delta \dot{\psi}_{N R_{j}}=\left(\Delta p_{N R_{j}} \sin \varphi_{N R}+\Delta q_{N R_{j}} \cos \varphi_{N R}\right) / \sin \theta_{N R}, \\
& \Delta \dot{\theta}_{N R_{j}}=\Delta p_{N R_{j}} \cos \varphi_{N R}-\Delta q_{N R_{j}} \sin \varphi_{N R}, \\
& \Delta \dot{\varphi}_{N R_{j}}=\Delta r_{N R_{j}}-\Delta \dot{\psi}_{N R_{j}} \cos \theta_{N R} .
\end{aligned}
$$

Here $\Delta p_{N R_{j}}, \Delta q_{N R_{j}}, \Delta r_{N R_{j}}$ represent the periodic part of each $\mathrm{j}$-th component of the non-rigid Earth angular velocity vector.

It should be also stated that the secular terms in the Euler angles in the case of the non-rigid Earth rotation and in the case of the rigid Earth rotation are the same, as follows from (4), (4a), (5).

This system is solved iteratively:

\section{1-st iteration}

In it

$\varphi_{N R}^{0}=\varphi_{R} \Rightarrow \Delta \dot{\theta}_{N R_{j}}^{1}=\Delta p_{N R_{j}} \cos \varphi_{N R}^{0}-\Delta q_{N R_{j}} \sin \varphi_{N R}^{0}$. By applying the "Cascade" method from $(8)$ follows $\theta_{N R}^{1} \Rightarrow \Delta \dot{\psi}_{N R_{j}}^{1}=\left(\Delta p_{N R_{j}} \sin \varphi_{N R}^{0}+\Delta q_{N R_{j}} \cos \varphi_{N R}^{0}\right) / \sin \theta_{N R}^{1} \Rightarrow \Delta \dot{\varphi}_{N R_{j}}^{1}=\Delta r_{N R_{j}}-\Delta \dot{\psi}_{N R_{j}}^{1} \cos \theta_{N R}^{1}$.

Besides, from $(8) \Rightarrow \psi_{N R}^{1}, \varphi_{N R}^{1}$. 


\section{2-nd iteration}

In it

$\psi_{N R}^{1}, \varphi_{N R}^{1} \Rightarrow \Delta \dot{\theta}_{N R_{j}}^{2}=\Delta p_{N R_{j}} \cos \varphi_{N R}^{1}-\Delta q_{N R_{j}} \sin \varphi_{N R}^{1}$. By applying the "Cascade" method from $(8)$ follows $\theta_{N R}^{2} \Rightarrow \Delta \dot{\psi}_{N R_{j}}^{2}=\left(\Delta p_{N R_{j}} \sin \varphi_{N R}^{1}+\Delta q_{N R_{j}} \cos \varphi_{N R}^{1}\right) / \sin \theta_{N R}^{2} \Rightarrow \Delta \dot{\varphi}_{N R_{j}}^{2}=\Delta r_{N R_{j}}-\Delta \dot{\psi}_{N R_{j}}^{2} \cos \theta_{N R}^{2}$.

Besides, from $(8) \Rightarrow \psi_{N R}^{2}, \varphi_{N R}^{2}$.

\section{n-th iteration}

In it

$\psi_{N R}^{n-1}, \varphi_{N R}^{n-1} \Rightarrow \Delta \dot{\theta}_{N R_{j}}^{n}=\Delta p_{N R_{j}} \cos \varphi_{N R}^{n-1}-\Delta q_{N R_{j}} \sin \varphi_{N R}^{n-1}$. By applying the "Cascade" method from (8) follows $\theta_{N R}^{n} \Rightarrow \Delta \dot{\psi}_{N R_{j}}^{n}=\left(\Delta p_{N R_{j}} \sin \varphi_{N R}^{n-1}+\Delta q_{N R_{j}} \cos \varphi_{N R}^{n-1}\right) / \sin \theta_{N R}^{n} \Rightarrow \Delta \dot{\varphi}_{N R_{j}}^{n}=\Delta r_{N R_{j}}-\Delta \dot{\psi}_{N R_{j}}^{n} \cos \theta_{N R}^{n}$. Besides, from $(8) \Rightarrow \psi_{N R}^{n}, \varphi_{N R}^{n}$.

The iterations are repeated when the absolute value of the difference between successive iterations exceeds some preassigned values $\varepsilon$.

\section{RESULTS}

The application of all considered transfer functions to S9000 and SMART97 results in the same amplitudes for the terms with a 18.6 year period. Table 1 shows the values of these amplitudes.

Table 1. The values of the amplitudes of the different solutions for the terms with an argument $\lambda_{3}+\mathrm{D}-\mathrm{F}$

\begin{tabular}{|r|c|c|c|c|c|c|}
\hline $\begin{array}{c}\text { Solution S9000 or SMART97 } \\
+ \text { transfer function of }\end{array}$ & $\begin{array}{c}\Psi(\sin ) \\
\mu \text { as }\end{array}$ & $\begin{array}{c}\Psi(\cos ) \\
\mu \text { as }\end{array}$ & $\begin{array}{c}\theta(\sin ) \\
\mu \text { as }\end{array}$ & $\begin{array}{c}\theta(\cos ) \\
\mu \text { as }\end{array}$ & $\begin{array}{c}\varphi(\sin ) \\
\text { Mas }\end{array}$ & $\begin{array}{c}\varphi(\cos ) \\
\mu \text { as }\end{array}$ \\
\hline Wahr (1981) & 17199072 & -431 & 35 & -9202843 & 15777192 & -395 \\
\hline Dehant and Defraigne (1997) & 17202786 & -431 & 35 & -9203936 & 15780599 & -396 \\
\hline Shirai and Fukushima (2001) & 17205158 & -2701 & -1269 & -9204660 & 15782776 & -2479 \\
\hline Bretagnon et al. (1999) & 17206626 & -3351 & -1486 & -9205148 & 15784122 & -3075 \\
\hline Mathews et al. (2002) & 17206664 & -3386 & -1505 & -9205211 & 15784185 & -3106 \\
\hline
\end{tabular}

Here $\lambda_{3}+\mathrm{D}-\mathrm{F}=\Omega+180^{\circ} ; \lambda_{3}$ is the mean longitude of the Earth; D is the difference between the mean longitudes of the Moon and the Sun; $\Omega$ is the mean longitude of the ascending node of the lunar orbit; $F$ is the mean argument of the Moon's latitude. 
Table 2. The comparison of the different solutions for the most important terms

\begin{tabular}{|c|c|c|c|c|r|r|r|}
\hline Solution & $\begin{array}{c}\text { Argu-ment, } \\
\text { Period (days) }\end{array}$ & $\begin{array}{c}\Psi(\sin ) \\
\mu \mathrm{as}\end{array}$ & $\begin{array}{c}\Psi(\cos ) \\
\mu \mathrm{as}\end{array}$ & $\begin{array}{c}\theta(\sin ) \\
\mu \mathrm{as}\end{array}$ & $\begin{array}{c}\theta(\cos ) \\
\mu \mathrm{as}\end{array}$ & $\begin{array}{c}\varphi(\sin ) \\
\mu \mathrm{as}\end{array}$ & $\begin{array}{c}\varphi(\mathrm{cos}) \\
\text { Mas }\end{array}$ \\
\hline SMN & $\lambda_{3}+\mathrm{D}-\mathrm{F}$ & 17206664.8552 & -3386.9864 & -1505.7856 & -9205211.3617 & 15784185.0624 & -3106.7674 \\
SN9000 & 6798.38 & 17206664.8552 & -3386.9864 & -1505.7856 & -9205211.3617 & 15784185.0624 & -3106.7674 \\
\hline SMN & $2 \lambda_{3}$ & -1318596.8582 & -748.6166 & -451.5369 & 572973.2222 & -1209767.5649 & -658.9659 \\
SN9000 & 182.62 & -1318596.6148 & -748.0244 & -451.1548 & 572973.2727 & -1209767.3333 & -658.4234 \\
\hline SMN & $2 \lambda_{3}+2 \mathrm{D}$ & -227640.1800 & 297.0399 & 135.7237 & 97843.9277 & -208855.8620 & 265.7426 \\
SN9000 & 13.66 & -227640.1593 & 297.0236 & 135.7144 & 97843.9181 & -208855.8434 & 265.7281 \\
\hline SMN & $2 \lambda_{3}+2 \mathrm{D}-2 \mathrm{~F}$ & 207463.2276 & -72.8532 & -28.7639 & -89756.6705 & 190409.8464 & -66.9362 \\
SN9000 & 3399.19 & 207463.2277 & -72.8533 & -28.7598 & -89756.6616 & 190409.8336 & -66.9358 \\
\hline SMN & $\lambda 3$ & -37360.1264 & -123079.8222 & 16721.8818 & 623.4780 & -34188.8930 & -112726.8142 \\
SN9000 & 365.24 & -37360.1264 & -123079.8222 & 16721.8818 & 623.4780 & -34188.8930 & -112726.8142 \\
\hline SMN & $3 \lambda_{3}$ & 11532.5978 & 50400.5095 & 21873.2512 & -5006.6994 & 10581.9069 & 46242.6261 \\
SN9000 & 121.75 & 11532.7794 & 50400.6781 & 21873.3218 & -5006.7717 & 10582.0752 & 46242.7801 \\
\hline
\end{tabular}

Here SMN=SMART97+MHB; SN9000=S9000+MHB; MHB- Mathews et al. (2002).

Table 2 gives the results of the comparison of SMN and SN9000 solutions for the most important terms. The geophysical models of SMN and SN9000 solutions include the same effects as in the model MHB2002 (the effects of Electromagnetic coupling, the Ocean effects, Mantle inelasticity effects, Atmospheric effects, Change in the global Earth dynamical flattening and in the core flattening).

It should be also stated that Table 1 and Table 2 describe SMART97 and S9000 solutions only in Dynamical case.

The differences between SN9000 and SMN, after the removal of the secular terms for the Newtonian case (Dynamical case) and for the relativistic one (Kinematical case), are depicted in Figure 1.

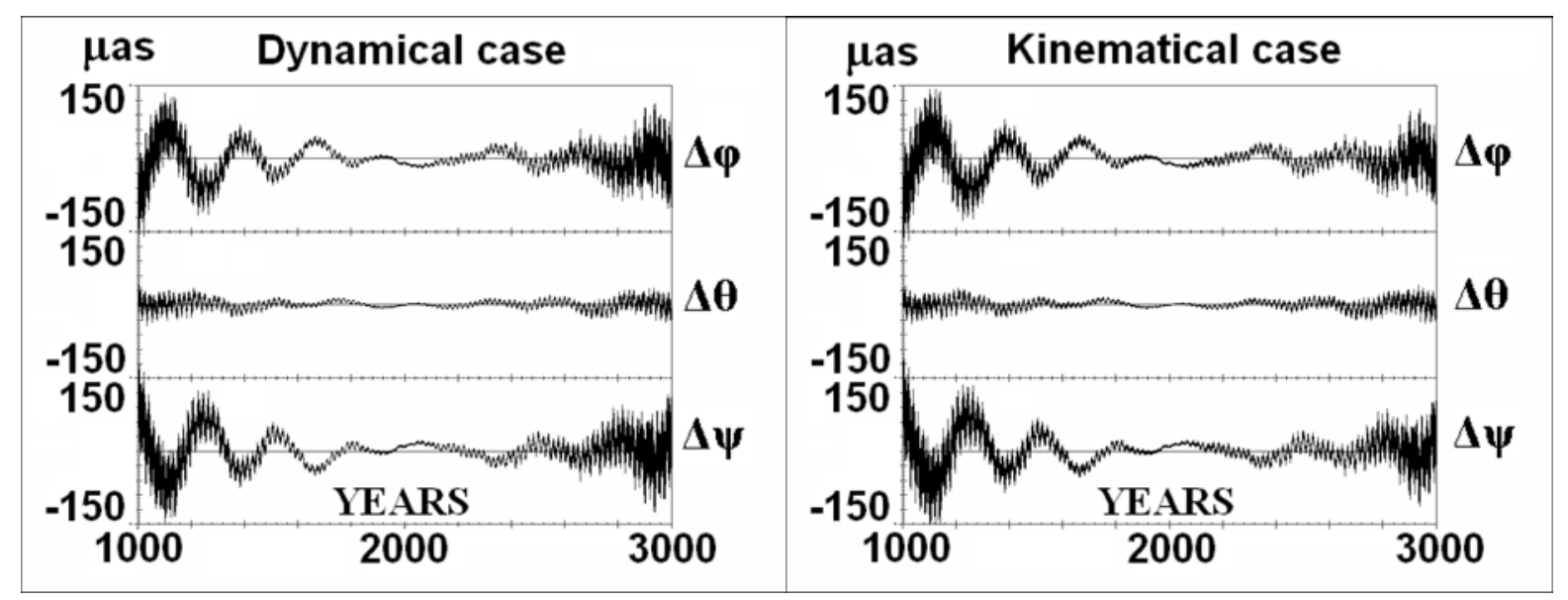

Fig.1. The differences between SN9000 and SMN after the removal of the secular terms

The differences between S9000 and SMART97 in Pashkevich et al. (2004) are the same as the differences between SN9000 and SMN. So, the Kinematical solutions for SN9000 and SMN are derived from the corresponding Dynamical solutions by adding identical 
expressions for the Geodetic corrections. After the removal of the secular trends the periodical residuals do not surpass $150 \mu$ as over 2000 time span years.

The Kinematical solution of the rigid Earth rotation is defined as the sum of the Dynamical solution of the rigid Earth rotation and the Geodetic corrections.

The Dynamical solution of the non-rigid Earth rotation is defined as the sum of the Dynamical solution of the rigid Earth rotation and the Transfer function.

The Kinematical solution of the non-rigid Earth rotation is defined as the sum of the Dynamical solution of the non-rigid Earth rotation and the Geodetic corrections.

\section{CONCLUSIONS}

1. The new exact expressions (3), containing the complex values, for the algorithm of Bretagnon et al. (1999), are obtained. The new formulas for the complex transformation form of the components of the rigid angular velocity vector to the components of the non-rigid angular velocity vector are obtained.

2. The new semi-analytical solution of the non-rigid Earth rotation SMN (SMART97 and MHB2002) is derived.

3. The high-precision non-rigid Earth rotation series SN9000, presented as functions of the three Euler angles, dynamically adequate to the ephemeris DE404/LE404 over 2000 years time span, are constructed.

\section{Acknowledgements}

The author highly appreciates very useful discussions with Prof. A. Brzeziński. The investigation was carried out at the Central (Pulkovo) Astronomical Observatory of Russian Academy of Science and the Space Research Centre of Polish Academy of Science, under a financial support within the program of cooperation between Polish and Russian Academies of Sciences (Theme No.31).

The author thanks the reviewer for very useful remarks and suggestions.

\section{REFERENCES}

Bretagnon P., Francou G. (1988) Planetary theories in rectangular and spherical variables, Astron. Astrophys., Vol. 202, pp. 309-315.

Bretagnon P., Francou G., Rocher P., Simon J.L. (1998) SMART97: a new solution for the rotation of the rigid Earth, Astron. Astrophys., Vol. 329, pp. 329-338.

Bretagnon P., Mathews P.M., Simon J.L. (1999) Non Rigid Earth Rotation, Proc. Journees Systèmes de Référence Spatio-Temporels 1999, eds. Soffel M., Capitaine N., Dresden 1999, pp. 73-76.

Brumberg V.A., Bretagnon P. (2000) Kinematical Relativistic Corrections for Earth's Rotation Parameters, Proc. of IAU Colloquium 180, eds. K. Johnston, D. McCarthy, B. Luzum, and G. Kaplan, U.S. Naval Observatory, pp. 293-302.

Dehant V. and Defraigne P. (1997) New transfer functions for nutations of a non-rigid Earth, J. Geophys. Res., 102, pp.27659-27688.

Mathews, P.M., Herring, T.A., and Buffett B.A. (2002) Modeling of nutation and precession: New nutation series for nonrigid Earth and insights into the Earth's Interior, J. Geophys. Res., 107, B4, 10.1029/2001JB000390. 
Eroshkin G.I., Pashkevich V.V., Brzezinski, A. (2004) Numerical analysis of the rigid Earth rotation with the quadruple precision, Artificial Satellites, Vol. 39, No. 4, pp. 291-304.

Pashkevich V. V. and Eroshkin G. I. (2004). Spectral analysis of the numerical theory of the rigid Earth rotation, Proc. Journees Systèmes de Référence Spatio-Temporels 2004, ed. Capitaine N., Paris 2004, pp. 82-87.

Pashkevich V.V. and Eroshkin G.I. (2005) Choice of the optimal spectral analysis scheme for the investigation of the Earth rotation problem, Proc. Journees Systèmes de Référence Spatio-Temporels 2005, eds. Brzezinski A., Capitaine N. and Kolaczek B., Warsaw 2005, pp. 105-109.

Pashkevich V. V., Eroshkin G. I. (2005) Application of the spectral analysis for the mathematical modelling of the rigid Earth rotation, Artificial Satellites, Vol. 40, No. 4, pp. 251-259.

Shirai T. and Fukushima T. (2001) Construction of a new forced nutation theory of the nonrigid Earth, The Astron. Journal, 121, pp.3270-3283.

Wahr J.M. (1981) The forced nutations of an elliptical, rotating, elastic and oceanless Earth, Geophys. J. R. Astron. Soc., 64, pp.705-727.

Received: 2008-06-03,

Reviewed: 2008-10-07,

Accepted: 2009-01-21. 